\title{
Polarized Light Emitting Diode by Long- Range Nanorod Self-Assembling on a Water Surface
}

\author{
Aurora Rizzo, * Concetta Nobile, Marco Mazzeo, Milena De Giorgi, Angela Fiore, Luigi Carbone, \\ Roberto Cingolani, Liberato Manna, and Giuseppe Gigli*
}

NNL, National Nanotechnology Laboratory of CNR-INFM, Distretto Tecnologico ISUFI, Dipartimento di Ingegneria dell'Innovazione, Università del Salento, via Arnesano Km 5 , I-73100 Lecce, Italy. ${ }^{\ddagger}$ Current address: Institute of Physical Chemistry, University of Mainz, Jakob-Welder-Weg 11, 55128 Mainz, Germany.

ABSTRACT We demonstrate a straightforward strategy to fabricate a multilayer inorganic/organic polarized light-emitting diode device based on highly ordered arrays of rod-shaped nanocrystals as the active species. We have developed a simple and effective method that allows colloidal $\mathrm{CdSe} / \mathrm{CdS}$ core/shell nanorods to be laterally aligned in smectic or nematic phases on the surface of water. A floating film of such ordered nanorods has been collected by a poly(dimethylsiloxane) (PDMS) stamp pad and transferred by contact printing onto previously evaporated organic layers. Thanks to the lateral nanorod alignment the as-prepared film exhibited strong polarized photoluminescence and it has been used as emissive layer in the polarized electroluminescent device.

KEYWORDS: nanorod self-assembly · nanorod alignment · nanorod LEDs · imprinting $\cdot$ polarized emission

${ }^{*}$ Address correspondence to aurora.rizzo@unisalento.it, giuseppe.gigli@unisalento.it.

Received for review January 21, 2009 and accepted May 06, 2009.

Published online May 15, 2009. 10.1021/nn900063m CCC: $\$ 40.75$

(c) 2009 American Chemical Society
$\mathrm{T}$ he ability to organize colloidal nanocrystals in ordered superstructures over large areas is of primary importance for the bottom-up fabrication of nanocrystal-based devices. Indeed, by controlling the spatial arrangement and the degree of order among the individual building blocks it is possible to obtain unique properties for a wide variety of application fields, such as solar cells, light-emitting diodes (LEDs), and polarized displays. ${ }^{1-4}$ Among these polarized LEDs have attracted the interest of both the scientific and industrial communities, as they can be used as a back-lighting source to improve the daytime contrast in liquid crystal display technology.

In the past few years, various techniques have been developed for this purpose. Essentially, they have been based on either promoting entropically driven self-assembly processes $s^{5-10}$ or deliberately driving the organization of nanoparticles by means of external perturbations, such as, for example, external electric fields, ${ }^{11-16}$ by the Langmuir-Blodgett technique ${ }^{17,18}$ or by exploiting the interfacial tension between two immiscible liquid phases. ${ }^{19}$ Nevertheless, despite the tremendous efforts de- voted up to now, the ability to produce ordered and large scale assemblies of shapecontrolled nanocrystals needs further advancements for their technological potential to be truly exploited in optoelectronic and photovoltaic devices. To date, efforts in this direction have remained confined to an isolated example of fabrication of LEDs emitting some degree of polarized light from layers of $\mathrm{CdSe} / \mathrm{CdS}$ core/ shell quantum rods aligned either in a stretched polymer matrix or by rubbing. ${ }^{4}$

In this paper, we report a novel deposition technique that enables the formation of smectic/nematic nanorod superstructure films and describes how to integrate them as an emissive layer into a hybrid organic/ inorganic LED. Since rod shaped nanoparticles show narrow band and linearly polarized emission, their alignment on a scale large enough to be compatible with the LED technology makes it possible to produce polarized electroluminescence.

Our technique consists of a two-step process. In the first step, a drop of colloidal nanorod solution is spread onto a water surface. During the solvent evaporation the nanorods self-assemble in an ordered film at the water/air interface. Subsequently, a poly(dimethylsiloxane) PDMS stamp pad is used to fish and transfer by contact printing the nanorod film from the water surface to a previously evaporated organic thin film. By exploiting the contact printing process it is possible to preserve the long-range order of the nanorod film and to fabricate a multilayer hybrid LED with strongly polarized emission along the alignment direction. Moreover the use of the contact printing technique allows for the independent processing of nanocrystal and organic material and, consequently, the fabrication of 
the hybrid device without any restriction in the organic material choice.

The technique proposed herein can be extended to different nanocrystalline materials and it could solve one of the critical problems related to the transfer of ordered nanocrystal assemblies on a desirable substrate for any kind of nanocrystal-based device fabrication.

\section{RESULTS AND DISCUSSION}

Deposition Technique. The shape anisotropy of nanorods makes their self-assembly into large scale ordered films extremely difficult to achieve unless the nanorods are characterized by narrow distributions of both diameters and lengths. Recent synthetic developments based on the seeded-growth approach ${ }^{16}$ have yielded $\mathrm{CdSe} / \mathrm{CdS}$ core/shell-type nanorods with narrow distributions of both lengths and diameters, which additionally emit wavelength-tunable, linearly polarized light with high efficiency. Here, we exploit the shape and size regularity of such type of nanorods to fabricate closepacked films of ordered arrays and to integrate them in a polarized light emitting device.

The sketch of the technique is reported in Figure 1. It consists in a two-step process, namely the selfassembly of a nanorod film at the water/air interface followed by its controlled transfer onto an organic layer for the integration of the nanorod film in a hybrid light emitting diode device. In the first step, a drop of a toluene solution containing $\mathrm{CdSe} / \mathrm{CdS}$ nanorods is spread over the surface of water in a glass beaker. The hydrophobic surfactant alkyl-chains coating on the nanorods make them float on the water surface. The solvent of the as-deposited nanorod solution is then allowed to evaporate slowly, in a toluene vapor-saturated environment at room temperature, over a period of $6-8 \mathrm{~h}$.

The vapor-saturated ambient is realized in a closed vessel that is partially filled with liquid toluene. In the second step, a PDMS flat stamp pad is brought in contact with the floating film. The hydrophobic silicone oligomers on the stamp surface interact strongly with the hydrophobic tails of the surfactant molecule that coat the nanorod surface, ${ }^{20,21}$ thereby enabling the transfer of the floating film from the water surface to the PDMS surface, while avoiding wetting of the stamp pad. The PDMS-supported nanorod film is then transferred via contact printing process onto the surface of an organic thin film (made of $4,4^{\prime}, N, N^{\prime}$-diphenylcarbazole, or CBP) that had been previously deposited by thermal evaporation on an indium tin oxide (ITO) glass substrate. ${ }^{21}$ The conductive ITO substrate was indeed preferred to facilitate the characterization of the nanorod-stamped organic film with scanning electron microscopy (SEM, see below). Both the low PDMS surface energy (21.6 $\mathrm{dyn} / \mathrm{cm}$ ) and the conformal contact of the stamp pad and the higher surface energy of the organic material allow an efficient transfer of the nanorod film. ${ }^{21,22}$ (a)

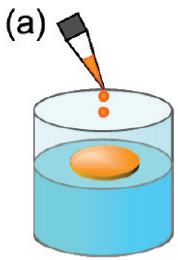

(b)

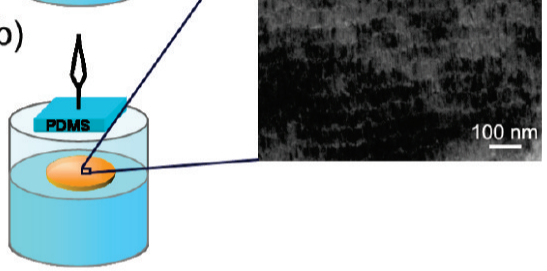

(c)

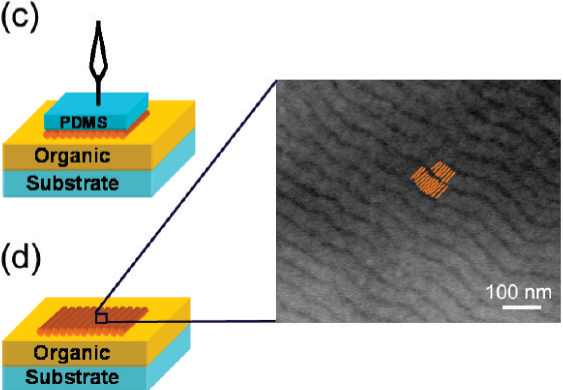

Figure 1. A sketch of the technique developed for the deposition of ordered nanorod arrays on organic layers. (a) A toluene solution of $\mathrm{CdSe} / \mathrm{CdS}$ nanorods is first spread on water. (b) After the solvent evaporation, the floating film is fished by means of a PDMS stamp pad. The zoom displays a transmission electron microscopy (TEM) image of a selfassembled nanorod film after being fished from the water surface by a carbon-coated TEM grid. The nanorods are arranged in ribbon-like structures in which they are joined side-by-side along their elongated $c$-axis, and thereby resembling the smectic phase of a liquid crystal. Some nanorods are highlighted in orange as a guide to the eye. (c) The inked PDMS is brought into conformal contact with the organic layer by applying a gentle pressure. (d) Finally the stamp is removed. The nanorod film is successfully transferred onto the organic surface. The zoom shows a scanning electron microscopy (SEM) image of the laterally aligned nanorod film after the transfer onto the organic layer. Some nanorods are highlighted in orange as a guide to the eye.

Nanorod Self-Assembly On a Water Surface. By accurately controlling the concentration of nanorods in the solution and by slowing down the solvent evaporation process it is possible to prepare films of close-packed nanorods floating on the surface of water organized in nematic and/or smectic phases. To check the degree of the nanorod alignment, after the solvent evaporation the floating film has been collected on a carbon-coated grid and then imaged by transmission electron microscopy (TEM) (see inset of Figure $1 \mathrm{~b}$ and also Figure $2 a-c)$.

We have observed that the experimental conditions of assembly in quasi-saturated closed environment (see Experimental Section), at ambient temperature and in the absence of external turbulences and convective flows on the water surface promote a high degree of nanorod lateral alignment. The lateral ordering under the present experimental conditions seems 

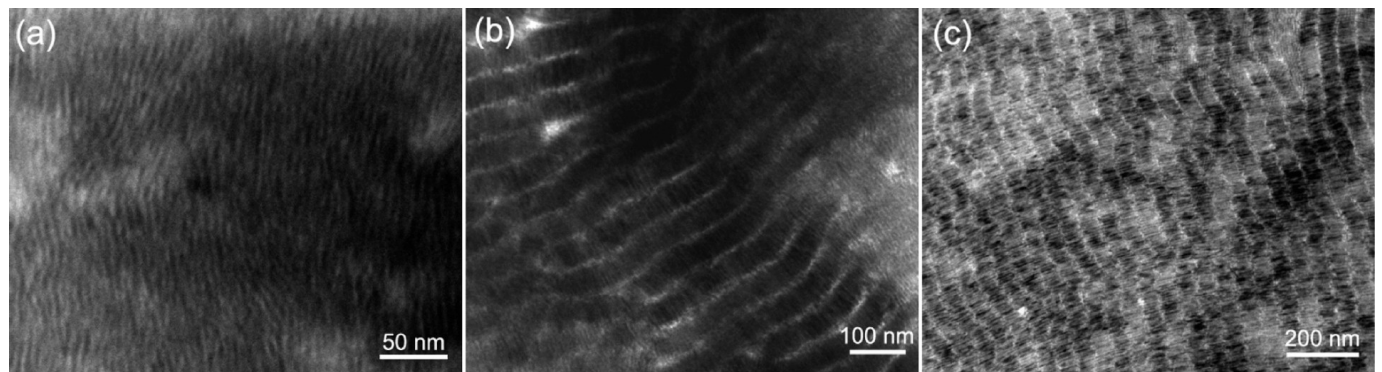

Figure 2. TEM pictures of the lateral aligned nanorod film. From the TEM images we observe the formation of either (a) nematic, (b) nematic and smectic, or (c) smectic order on the same nanorod floating film.

to be favored to the vertical arrangement of nanorods as we have reported in a precedent work, in which the assembly process was carried out on the water surface under different conditions (no vapor saturation and higher water temperatures, up to $\left.60^{\circ} \mathrm{C}\right) .^{16}$

The tendency toward self-organization of monodisperse colloidal nanorods can be understood in terms of surface tension balance. When a small volume of a concentrated organic solution of nanorods is spread onto a water surface, the slow and uniform solvent evaporation leads to a continuous increase in the concentration of nanocrystals in the remaining droplet. As soon as the solvent evaporates, hydrophobic and dipolar interactions among the surfactant-coated nanorods are progressively enhanced, due to the gradually increasing concentration of nanorods in the organic phase, that is, shortening of the nanorod interparticle distance. Such intermolecular forces induce segregation of the nanorods in close-packed lateral ribbon superstructures or rod/rafts aggregates, as a means of decreasing the overall surface and interfacial energy of the system. ${ }^{17,23}$

According to the Onsager hard-rod model ${ }^{24}$ the parallel positional order is favorable because it increases the translational entropy by minimizing the excluded volume from the center of mass of the approaching rods, and therefore it predicts that rod-shaped objects will self-organize into nematic phase. Additionally, Frenkel et al. ${ }^{25}$ presented numerical evidence that for hardrod fluids with both translational and orientational freedom can form a thermodynamically stable smectic phase. Maeda and Maeda ${ }^{26,27}$ suggested the formation of rod clusters aligned side-by-side already in solution at high concentrations. The increase in the particle/ volume fraction restricts the Brownian motions and in particular the rotational motions, leading to the formation of ribbon-like structures. The length and the persistence time of the ribbons in solution are proportional to the concentration and depend on the nanorod aspect ratio. Furthermore cadmium chalcogenide nanorods present a permanent dipole moment along the long axis due to the noncentrosymmetric wurtzite lattice structure. ${ }^{12,28}$ Talapin et al. $^{9}$ and Bunge et al. ${ }^{29}$ reported that the attractive dipole-dipole interaction between adjacent nanorods could lead to antiparallel side-by-side pairing of dipole moments and facilitate the formation of smectic phases.

The formation of a smectic phase can be additionally explained by considering that the basal polar facets of the nanorods are less efficiently passivated by the surfactants relative to the other longitudinal facets, hence residual, uncompensated surface charges might be localized at the nanorod tips. Therefore the lateral nanorod alignment allows in principle for the maximization of both hydrophobic side-by-side and electrostatic end-by-end interactions among the packed nanorods. $^{23}$

Nanorod Film on Organic Layer Characterization. The quality of the nanorod film transferred by contact printing onto the organic layer was verified by scanning electron microscopy (SEM) imaging. Figure 3 shows SEM images of different regions of the nanorod film on the organic layer at different scanning areas. The rods were aligned either in nematic or smectic phases over areas on the scale of hundreds square micrometers and the order was preserved after the transfer.

Polarized emission from the aligned regions was collected by a conventional photoluminescence setup in the epilayer configuration and in ambient conditions at room temperature. The apparatus is depicted in Figure $4 \mathrm{a}$ and consists of a circularly polarized $\mathrm{He}-\mathrm{Cd}$ laser $(\lambda$ $=325 \mathrm{~nm}$ ), an objective lens (LMU-20X-325, 20× magnification, NA $=0.4$ ), a $0.33 \mathrm{~m}$ monochromator (Jobin Yvon TRIAX320, f/\# = 4.1 with a grating $=600 \mathrm{~L} / \mathrm{mm}$ blazed at $500 \mathrm{~nm}$ ) equipped with an Andor CCD camera (DV420-UV thermoelectrically cooled down to -70 $\left.{ }^{\circ} \mathrm{C}\right)$. The sample is excited by the laser at $325 \mathrm{~nm}$. The polarized emission was analyzed with a linear polarizer in combination with an achromatic $\lambda$-half plate, placed in front of the monochromator to correct the polarization-dependent response of both the monochromator and the detector. By using a slit width of the monochromator of $500 \mathrm{~nm}$ and an integration time of $0.5 \mathrm{~s}$ we observed high photoluminescence $(\mathrm{PL})$ intensity by the oriented nanorod films in the polarization direction parallel to the alignment orientation, in agreement with earlier polarization measurements on single nanorods ${ }^{30}$ and on ordered nanorod arrays. ${ }^{16,31}$

In Figure $4 \mathrm{~b}$ the PL spectra along two perpendicular polarization directions are reported, which 

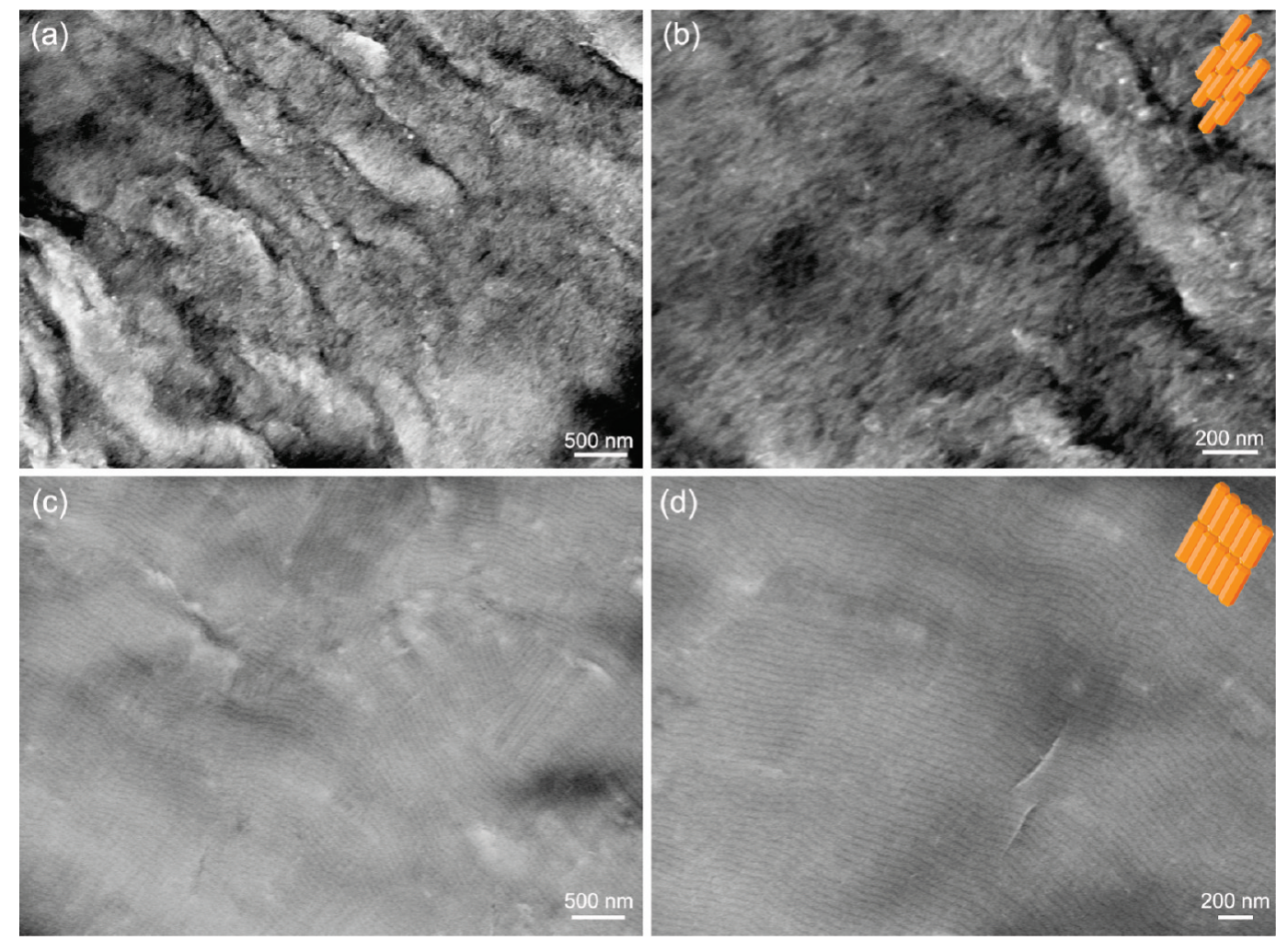

Figure 3. SEM characterization of the lateral aligned nanorod film. Low and high magnification SEM images of $(a, b)$ nematic and (c, d) smectic regions of the nanorod film transferred on the organic semiconductor CBP layer. In the nematic phase the nanorods are longitudinally aligned along a common direction, that is, orientational ordering, but do not present a positional order, while in the smectic phase they are arranged with both positional and orientational order.
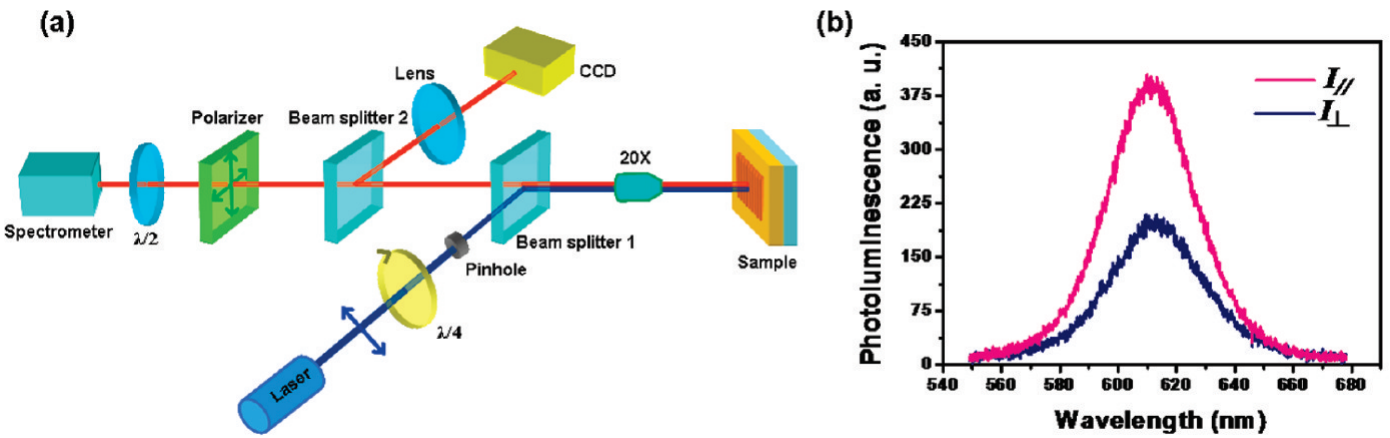

(c)

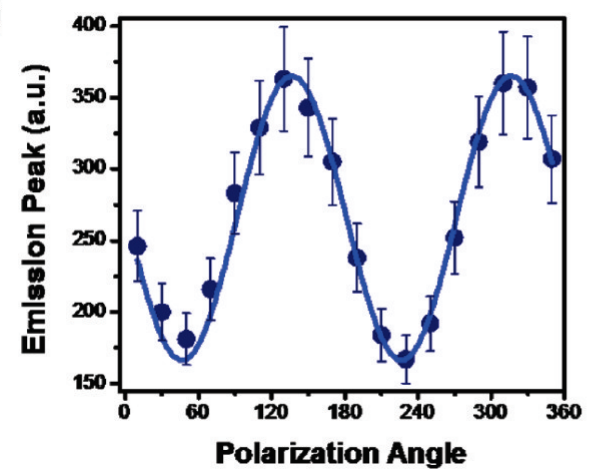

(d)

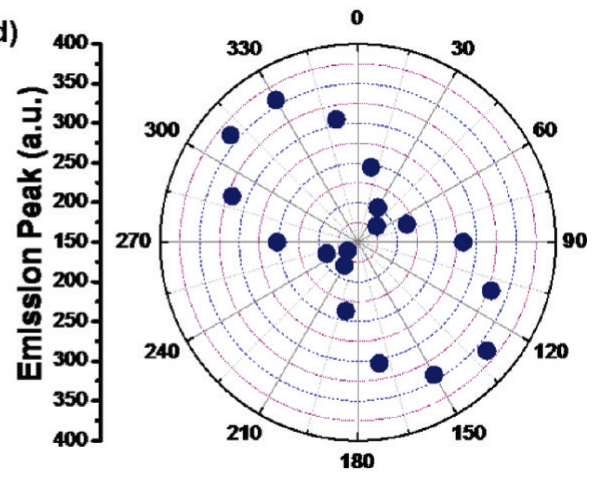

Figure 4. (a) A sketch of the experimental setup used in the optical measurements. (b) PL spectra recorded in two perpendicular polarization directions: $I_{\|}$parallel and $I_{\perp}$ perpendicular to the nanorod alignment direction. (c) PL peak intensity dependence on the polarization angle fitted to cosinusoidal function (blue line) and (d) the corresponding polar diagram. 

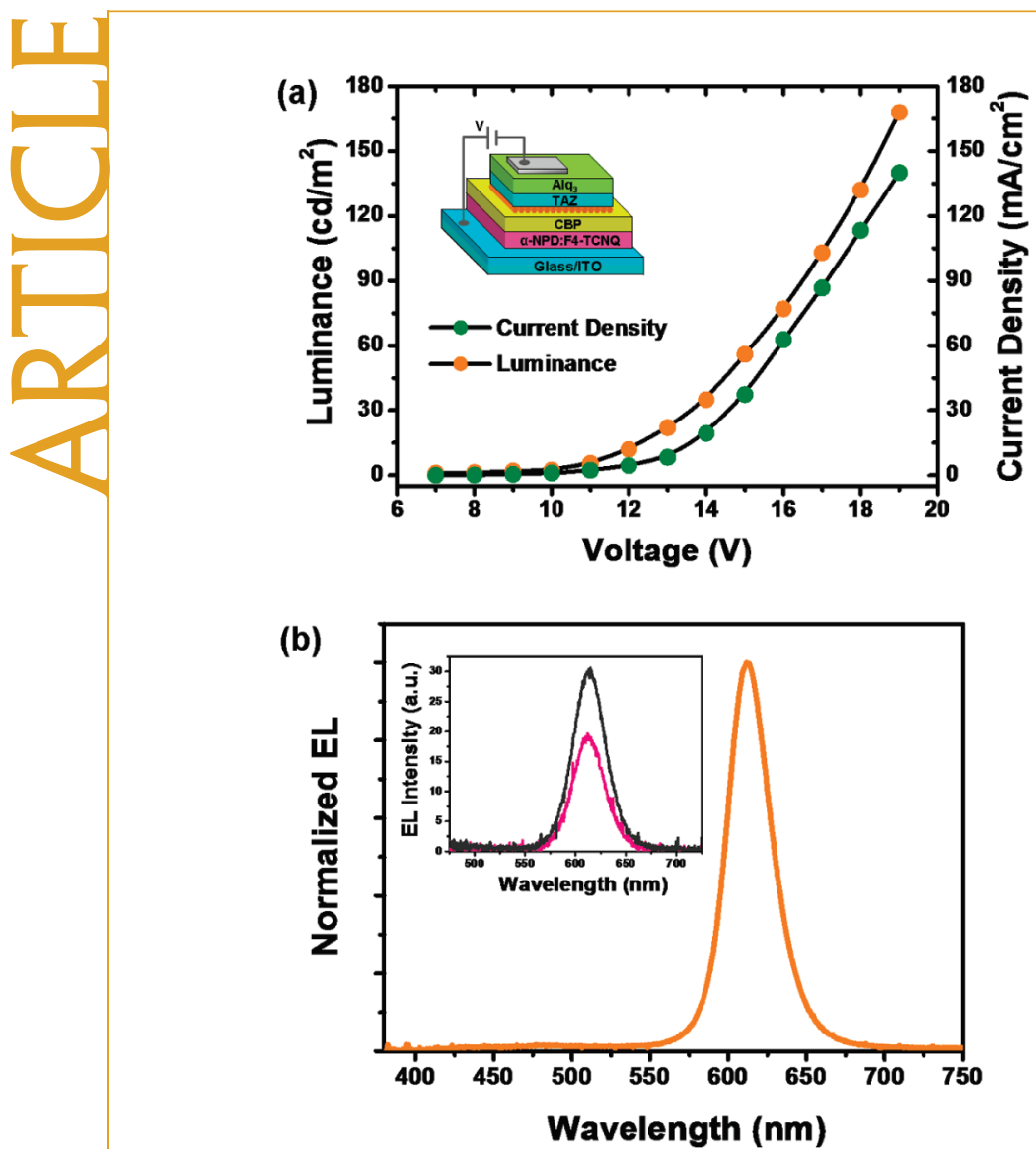

Figure 5. (a) Current density and luminance as a function of the applied voltage. The inset shows a sketch of the hybrid nanorod/ organic LED structure. (b) Normalized electroluminescence (EL) spectrum of the nanorod LED at a $12 \mathrm{~V}$ applied bias. In the inset the electroluminescence spectra for two orthogonal directions are reported.

correspond to the maximum and minimum intensity. The PL intensity dependence on the polarization angle was fitted with by cosinusoidal function (Figure 4c) and the polar diagram is reported in Figure $4 \mathrm{~d}$. The degree of linear polarization is expressed as: $\left(I_{\|}-I_{\perp}\right) /\left(l_{\|}+I_{\perp}\right)$ where $I_{\|}$and $I_{\perp}$ represent the emission intensity parallel and perpendicular to the nanorod alignment direction, respectively. We found a maximum polarization ratio of 0.34 .

Polarized Light Emitting Device. The aforementioned technique for transferring the floating nanorod film onto an organic layer can be exploited for the fabrication of hybrid LEDs. To this aim, we have fabricated a multilayer organic/inorganic device structure in which the nanorod film acts as the emissive layer. The device structure (see the inset of Figure $5 \mathrm{a}$ ) consists of a thermally evaporated $N, N^{\prime}$-bis(naphthalen-1-yl)- $N, N^{\prime}$ bis(phenyl)benzidine ( $\alpha$-NPD) hole injection layer (HIL) doped with 2,3,5,6-tetrafluoro-7,7,8,8-tetracyanoquinodimethane (F4-TCNQ) and a CBP hole transporting layer (HTL). The nanorod active layer is deposited onto the organic substrate by means of the stamping technique reported above. The 3-(4-biphenylyl)-4phenyl-5-t-butylphenyl-1,2,4-triazole (TAZ) hole blocking layer $(\mathrm{HBL})$, the tris(8-(hydroxyl-quinoline) alumi- num $\left(\mathrm{Alq}_{3}\right)$ electron transporting layer (ETL), and LiF/AI electrodes are then evaporated after the nanorod deposition.

In Figure 5a current-voltage and luminance-voltage characteristics of the nanorod device are reported. The maximum brightness of 170 $\mathrm{cd} / \mathrm{m}^{2}$ was reached at $19 \mathrm{~V}$ and at $140 \mathrm{~mA} / \mathrm{cm}^{2}$, and the maximum current efficiency of $0.24 \mathrm{~cd} / \mathrm{A}$ was reached at $12 \mathrm{~V}$. The electroluminescence (EL) spectrum in Figure $5 \mathrm{~b}$ demonstrates that the emitted light comes from the nanorods. The EL spectra for two orthogonal polarization directions are reported in the inset of Figure $5 b$, and show a trend that is consistent with the PL polarization measurements of Figure 4.

In this type of device structure the exciton formation in the nanorod layer can occur either via charge trapping or via Förster energy transfer process from the organic material. ${ }^{32,33}$ In the charge trapping process electrons are easily transferred into the nanocrystal layer due to the nonexisting barrier at the $\mathrm{Alq}_{3} / \mathrm{Taz} /$ nanorod interface, thus they recombine with the holes injected from the organic under-layers. In the proposed structure the holes injection has been improved by $\mathrm{p}$-doping the $\alpha$-NPD matrix with the strong electron acceptor F4TCNQ. The F4-TCNQ molecules remove electrons from the $\alpha$-NPD highest occupied molecular orbital (HOMO) states and hence generate holes. ${ }^{34}$ Moreover the use of a TAZ HBL avoids the transfer of holes and excitons into the $\mathrm{Alq}_{3} \mathrm{ETL}$ and consequently inhibits the emission from this layer. Resonant Förster energy transfer has been demonstrated in similar hybrid LEDs. ${ }^{32,33}$ Excitons in the organic molecules, such as CBP and $\mathrm{Alq}_{3}$, are then transferred by dipole-dipole interaction to the lower energy nanocrystal sites. According with the literature in our device structure, resonant exciton energy transfer can probably occur at the interface between nanocrystals and $\mathrm{Alq}_{3}$ in the voids imperfections of TAZ film or CBP, favoring the excitation and the radiative recombination in the red emitting nanorods. ${ }^{33}$

The maximum brightness of $170 \mathrm{~cd} / \mathrm{m}^{2}$ is almost double if compared to a similar structure recently reported by our group for red emitting quantum dots (QDs) deposited by a similar microcontact printing technique. ${ }^{21}$ Nevertheless the device performances are lower if compared to the polarized quantum rod LED reported by Hikmet et al., ${ }^{4}$ for which a maximum luminance of $450 \mathrm{~cd} / \mathrm{m}^{2}$ and current efficiency of $0.65 \mathrm{Cd} / \mathrm{A}$ were reported. Differences in the device performances can be attributed either to the nanocrystal deposition technique or to differences in the properties and luminescence efficiencies of nanocrystal samples. As a matter of fact, higher device performances have been demonstrated for hybrid LEDs in which microcontact printing has been combined with spin-coating for QD deposition due to a better film formation and thickness control. ${ }^{35,36}$ Moreover Sun et al. demonstrated that nanocrystal based LED performances depend strongly 
on the QD luminescence efficiency, the covering surfactant, and layer thickness and properties. ${ }^{37}$

\section{CONCLUSION}

In conclusion, the results presented here demonstrate the possibility to form films of $\mathrm{CdSe} / \mathrm{CdS}$ nanorods that are assembled in smectic and nematic phases of nanorods over large areas. Polarized photoluminescence measurements showed high photoemission that was linearly polarized along the nanorod alignment direction (with a polarization factor up to $34 \%$ ). These films were successfully transferred onto organic layers by microcontact printing and were then integrated in a multilayer hybrid LED structure. The film transfer technique reported here represents a proof of concept and a promising approach to solve one of the critical problems related to the fabrication of nanocrystal-based devices, that is, the transfer of large ordered assemblies of nanocrystals to a different substrate for subsequent incorporation in hybrid LEDs as well as in nanocrystalbased optoelectronic devices and solar cells.

\section{EXPERIMENTAL SECTION}

The synthesis and the optical properties of the $\mathrm{CdSe} / \mathrm{CdS}$ core/shell nanorods used in this work can be found in ref 16 .

PDMS stamps were created by curing Sylgard 184 (a two ingredient elastomer kit purchased from Down Corning) on the top of a blank silicon master.

Nanorod Film Preparation. A $30 \mathrm{~nm}$ layer of $4,4^{\prime}, N, N^{\prime}-$ diphenylcarbazole (CBP) was thermal evaporated at a pressure of $2.0 \times 10^{-6} \mathrm{mbar}$ on ITO substrates. A $400 \mu \mathrm{L}$ drop of a toluene solution containing $\mathrm{CdSe} / \mathrm{CdS}$ nanorods $\left(\sim 10^{-8} \mathrm{M}\right)$ was spread over the surface of water in a glass beaker. The solvent of the as-deposited nanorod solution was then allowed to evaporate slowly, in a toluene vapor-saturated environment at room temperature, over a period of $6-8 \mathrm{~h}$. The quasi-saturated environment was realized by partially filling a desiccator with toluene. A small hole on the top of the desiccator was left opened. After the solvent evaporation the nanorod floating film was collected by a flat PDMS stamp and then it was transferred via a contact printing process onto the surface of the CBP organic thin film.

The SEM imaging was performed by the RAITH 150 EBL instrument at an accelerating voltage of $5 \mathrm{kV}$.

For the transmission electron microscopy (TEM) characterization the nanorod floating film was collected on a carbon-coated grid and it was imaged by the Jeol Jem 1011 microscope operating at an accelerating voltage of $100 \mathrm{kV}$.

Polarization Measurements. The polarized photoluminescence setup consisted of a circularly polarized $\mathrm{He}-\mathrm{Cd}$ laser $(\lambda=325$ $\mathrm{nm})$, an objective lens (LMU-20X-325, 20× magnification, $\mathrm{NA}=0.4$ ), a $0.33 \mathrm{~m}$ monochromator (Jobin Yvon TRIAX320, $\mathrm{f} / \#=$ 4.1 with a grating $=600 \mathrm{~L} / \mathrm{mm}$ blazed at $500 \mathrm{~nm}$ ) equipped with an Andor CCD camera (DV420-UV thermoelectrically cooled down to $-70^{\circ} \mathrm{C}$ ). The sample was excited by the laser at 325 $\mathrm{nm}$. To correct the polarization-dependent response of both the monochromator and the detector, a polarizer and a half wave plate were used. The correct alignment of their axis was verified by using a polarized laser.

Before starting the measurements the distance between the objective and the target was optimized by using a reference sample of nonaligned nanorods on a $\mathrm{SiC}$ substrate. On the substrate, $8 \mu \mathrm{m}$ wide stripes with a $3 \mu \mathrm{m}$ pitch were realized by electron beam lithography (EBL) and measured by SEM. A beam splitter placed between the sample and the monochromator was used to image both the sample and the laser spot on the CCD camera. The optical set up was accurately aligned to get the best image of the sample and the minimum laser spot. During the alignment, we verified that the objective was properly filled by the laser. Moreover, we have looked at the image on the CCD of both the laser focused on the sample and the photoluminescence signal. The objective mounted on a translation stage was moved to minimize the spot size of the image on the CCD array and to simultaneously maximize the intensity of both the laser and the PL signal. During the measurements the polarizer and the half-wave plate were rotated of $\theta$ and $\theta / 2$, respectively. We also verified that small angle deviation of the half-wave plate, $\Delta \theta$ $\approx 1^{\circ}$, from a fixed $\theta / 2$ value did not induce changes in the detected PL intensity. Polarization measurements performed on the reference sample did not show any significant polarization of the fluorescence emission. Finally the reference sample was replaced with the aligned nanorod sample and the focus was adjusted by means of a micrometer translation stage.

Hybrid Electroluminescent Device. The $40 \mathrm{~nm} \mathrm{HIL}$ and $30 \mathrm{~nm} \mathrm{HTL}$, respectively, $N, N^{\prime}$-bis(naphthalen-1-yl)- $N, N^{\prime}$ -

bis(phenyl)benzidine ( $\alpha$-NPD) doped with 2,3,5,6-tetrafluoro7,7,8,8-tetracyano-quinodimethane (F4-TCNQ) and CBP were evaporated in sequence on cleaned and oxygen-plasma-treated indium tin oxide ITO substrates. Then a nanorod film layer was deposited on the organic materials by $\mu \mathrm{CP}$. Finally a $15 \mathrm{~nm}$ holeblocking, 3-(4-biphenylyl)-4-phenyl-5-t-butylphenyl-1,2,4triazole (TAZ), and $20 \mathrm{~nm}$ electron- transporting, tris(8-(hydroxylquinoline) aluminum $\left(\mathrm{Alq}_{3}\right)$, layers were deposited by thermal evaporation. All the organic materials were thermal evaporated at a rate of $1 \AA / \mathrm{s}$ at a pressure of $2 \times 10^{-6} \mathrm{mbar}$. The $0.5 \mathrm{~nm}$ lithium fluoride (LiF) and $200 \mathrm{~nm}$ aluminum (Al) electrodes were evaporated through a contact shadow mask at a pressure of 4 $\times 10^{-6} \mathrm{mbar}$.

The electroluminescence (EL) spectra and luminance were measured by using an OL-770 spectroradiometer (Optronic Laboratories). Polarization EL measurements were performed by fitting the spectroradiometer with a polarizer.

Current-voltage characteristic were measured by using a Keithley 2400 source measurement unit. All the measurements were performed at room temperature in air without encapsulation.

\section{REFERENCES AND NOTES}

1. Huynh, W. U.; Dittmer, J. J.; Alivisatos, A. P. Hybrid Nanorod-Polymer Solar Cells. Science 2002, 295 (5564), 2425-2427.

2. Gur, I.; Fromer, N. A.; Geier, M. L.; Alivisatos, A. P. Air-Stable All-Inorganic Nanocrystal Solar Cells Processed from Solution. Science 2005, 310, 462-465.

3. Coe-Sullivan, S.; Steckel, J. S.; Woo, W. K.; Bawendi, M. G.; Bulovic, V. Large-Area Ordered Quantum-Dot Monolayers via Phase Separation During Spin-Casting. Adv. Funct. Mater. 2005, 15 (7), 1117-1124.

4. Hikmet, R. A. M.; Chin, P. T. K.; Talapin, D. V.; Weller, H. Polarized-Light-Emitting Quantum-Rod Diodes. Adv. Mater. 2005, 17 (11), 1436-1439.

5. Rogach, A. L.; Talapin, D. V.; Shevchenko, E. V.; Kornowski, A.; Haas ${ }^{\mathrm{e}}$, M.; Weller, H. Organization of Matter on Different Size Scales: Monodisperse Nanocrystals and Their Superstructures. Adv. Funct. Mater. 2002, 12 (10), 653-664.

6. Shevchenko, E. V.; Talapin, D. V.; Kotov, N. A.; O'Brien, S.; Murray, C. B. Structural Diversity in Binary Nanoparticle Superlattices. Nature 2006, 439 (7072), 55-59.

7. Li, L. S.; Alivisatos, A. P. Semiconductor Nanorod Liquid Crystals and their Assembly on a Substrate. Adv. Mater. 2003, 15 (5), 408-411.

8. Li, L. S.; Walda, J.; Manna, L.; Alivisatos, A. P. Semiconductor Nanorod Liquid Crystals. Nano Lett. 2002, 2 (6), 557-560. 
9. Talapin, D. V.; Shevchenko, E. V.; Murray, C. B.; Kornowski, A.; Forster, S.; Weller, H. CdSe and CdSe/CdS Nanorod Solids. J. Am. Chem. Soc. 2004, 126 (40), 12984-12988.

10. Querner, C.; Fischbein, M. D.; Heiney, P. A.; Drndić, M. Millimeter-Scale Assembly of CdSe Nanorods into Smectic Superstructures by Solvent Drying Kinetics. Adv. Mater. 2008, 20 (12), 2308-2314.

11. Harnack, O.; Pacholski, C.; Weller, H.; Yasuda, A.; Wessels, J. M. Rectifying Behavior of Electrically Aligned $\mathrm{ZnO}$ Nanorods. Nano Lett. 2003, 3 (8), 1097-1101.

12. Ryan, K. M.; Mastroianni, A.; Stancil, K. A.; Liu, H. T.; Alivisatos, A. P. Electric-Field-Assisted Assembly of Perpendicularly Oriented Nanorod Superlattices. Nano Lett. 2006, 6, 1479-1482.

13. Gupta, S.; Zhang, Q. L.; Emrick, T.; Russell, T. P. “SelfCorralling" Nanorods Under an Applied Electric Field. Nano Lett. 2006, 6 (9), 2066-2069.

14. Hu, Z. H.; Fischbein, M. D.; Querner, C.; Drndic, M. ElectricField-Driven Accumulation and Alignment of $\mathrm{CdSe}$ and CdTe Nanorods in Nanoscale Devices. Nano Lett. 2006, 6 (11), 2585-2591.

15. Nobile, C.; Fonoberov, V. A.; Kudera, S.; Della Torre, A.; Ruffino, A.; Chilla, G.; Kipp, T.; Heitmann, D.; Manna, L.; Cingolani, R. Confined Optical Phonon Modes in Aligned Nanorod Arrays Detected by Resonant Inelastic Light Scattering. Nano Lett. 2007, 7 (2), 476-479.

16. Carbone, L.; Nobile, C.; De Giorgi, M.; Sala, F. D.; Morello, G.; Pompa, P.; Hytch, M.; Snoeck, E.; Fiore, A.; Franchini, I. R.; et al. Synthesis and Micrometer-Scale Assembly of Colloidal CdSe/CdS Nanorods Prepared by a Seeded Growth Approach. Nano Lett. 2007, 7, 2942-2950.

17. Kim, F.; Kwan, S.; Akana, J.; Yang, P. D. Langmuir-Blodgett Nanorod Assembly. J. Am. Chem. Soc. 2001, 123 (18), 4360-4361.

18. Yang, P. D.; Kim, F. Langmuir-Blodgett Assembly of OneDimensional Nanostructures. Chemphyschem 2002, 3 (6), 503-506.

19. Wang, Y.; Guo, S.; Chen, H.; Wang, E. Facile Fabrication of Large Area of Aggregated Gold Nanorods Film for Efficient Surface-Enhanced Raman Scattering. J. Colloid Interface Sci. 2008, 318, 82-87.

20. Schmid, H.; Wolf, H.; Allenspach, R.; Riel, H.; Karg, S.; Michel, B.; Delamarche, E. Preparation of Metallic Films on Elastomeric Stamps and their Application for Contact Processing and Contact Printing. Adv. Funct. Mater. 2003 , $13(2), 145-153$.

21. Rizzo, A.; Mazzeo, M.; Palumbo, M.; Lerario, G.; D’Amone, S.; Cingolani, R.; Gigli, G. Hybrid Light-Emitting Diodes from Microcontact-Printing Double-Transfer of Colloidal Semiconductor CdSe/ZnS Quantum Dots onto Organic Layers. Adv. Mater. 2008, 20 (10), 1886-1891.

22. Xia, Y. N.; Whitesides, G. M. Soft Lithography. Annu. Rev. Mater. Sci. 1998, 28, 153-184.

23. Ghezelbash, A.; Koo, B.; Korgel, B. A. Self-Assembled Stripe Patterns of CdS Nanorods. Nano Lett. 2006, 6 (8), 1832-1836.

24. Onsager, L. The Effects of Shape on the Interaction of Colloidal Particles. Ann. N.Y. Acad. Sci. 1949, 51, 627-659.

25. Frenkel, D.; Lekkerkerker, H. N. W.; Stroobants, A. Thermodinamic Stability of a Smectic Phase in a System of Hard-Rods. Nature 1988, 332, 822-823.

26. Maeda, H.; Maeda, Y. Liquid Crystal Formation in Suspensions of Hard Rodlike Colloidal Particles: Direct Observation of Particle Arrangement and Self-Ordering Behavior. Phys. Rev. Lett. 2003, 90, 018303.

27. Maeda, H.; Maeda, Y. Direct Observation of Brownian Dynamics of Hard Colloidal Nanorods. Nano Lett. 2007, 7, 3329-3335.

28. Li, L. S.; Alivisatos, A. P. Origin and Scaling of the Permanent Dipole Moment in CdSe Nanorods. Phys. Rev. Lett. 2003, 90, 097402.

29. Bunge, S. D.; Krueger, K. M.; Boyle, T. J.; Rodriguez, M. A.; Headley, T. J.; Colvin, V. L. Growth and Morphology of Cadmium Chalcogenides: The Synthesis of Nanorods,
Tetrapods, and Spheres from $\mathrm{CdO}$ and $\mathrm{Cd}\left(\mathrm{O}_{2} \mathrm{CCH}_{3}\right)_{2}$. J. Mater. Chem. 2003, 13 (7), 1705-1709.

30. Hu, J. T.; Li, L. S.; Yang, W. D.; Manna, L.; Wang, L. W.; Alivisatos, A. P. Linearly Polarized Emission from Colloidal Semiconductor Quantum Rods. Science 2001, 292 (5524), 2060-2063.

31. Talapin, D. V.; Koeppe, R.; Gotzinger, S.; Kornowski, A.; Lupton, J. M.; Rogach, A. L.; Benson, O.; Feldmann, J.; Weller, H. Highly Emissive Colloidal CdSe/CdS Heterostructures of Mixed Dimensionality. Nano Lett. 2003, 3 (12), 1677-1681.

32. Li, Y. Q.; Rizzo, A.; Cingolani, R.; Gigli, G. Bright White-LightEmitting Device from Ternary Nanocrystal Composites. Adv. Mater. 2006, 18 (19), 2545-2548.

33. Anikeeva, P. O.; Halpert, J. E.; Bawendi, M. G.; Bulovic, V. Electroluminescence from a Mixed Red-Green-Blue Colloidal Quantum Dot Monolayer. Nano Lett. 2007, 7 (8), 2196-2200.

34. Walzer, K.; Maennig, B.; Pfeiffer, M.; Leo, K. Highly Efficient Organic Devices Based on Electrically Doped Transport Layers. Chem. Rev. 2007, 107 (4), 1233-1271.

35. Rizzo, A.; Mazzeo, M.; Biasiucci, M.; Cingolani, R.; Gigli, G. White Electroluminescence from a Microcontact-PrintingDeposited CdSe/ZnS Colloidal Quantum-Dot Monolayer. Small 2008, 4 (12), 2143-2147.

36. Kim, L. A.; Anikeeva, P. O.; Coe-Sullivan, S. A.; Steckel, J. S.; Bawendi, M. G.; Bulovic, V. Contact Printing of Quantum Dot Light-Emitting Devices. Nano Lett. 2008, 8 (12), $4513-$ 4517.

37. Sun, Q. J.; Wang, Y. A.; Li, L. S.; Wang, D. Y.; Zhu, T.; Xu, J.; Yang, C. H.; Li, Y. F. Bright, Multicoloured Light-Emitting Diodes Based on Quantum Dots. Nat. Photonics 2007, 1, 717-722. 